\title{
MED12, TERT promoter and RBM15 mutations in primary and recurrent phyllodes tumours
}

\author{
Diego A Garcia-Dios ${ }^{1}$, Dina Levi ${ }^{1}$, Vandna Shah ${ }^{1}$, Cheryl Gillett ${ }^{1}$, Michael A Simpson ${ }^{2}$, Andrew Hanby ${ }^{3}$, \\ Ian Tomlinson ${ }^{4}$ and Elinor J Sawyer ${ }^{\star}, 1$ \\ ${ }^{1}$ School of Cancer and Pharmaceutical Sciences, Guy's Hospital, King's College London, London SE1 9RT, UK; ${ }^{2}$ Medical and \\ Molecular Genetics, Guy's Hospital, King's College London, London, UK; ${ }^{3}$ Leeds Institute of Cancer and Pathology, Cancer \\ Genetics Building, St James's University Hospital, Beckett Street, Leeds LS9 7TF, UK and Institute of Cancer and Genomic \\ Sciences, University of Birmingham, Edgbaston, Birmingham B15 2TT, UK
}

Background: MED12 and TERT promoter mutations have been shown to be the most common somatic mutations in phyllodes tumours (PTs). The aims of this study were to determine the frequency of these mutations in recurrent PTs, assess whether TERT promoter mutations could be helpful in distinguishing fibroadenomas (FAs) from PTs and identify novel mutations that may be driving malignant progression.

Methods: MED12 and the TERT promoter were Sanger sequenced in 75 primary PTs, 21 recurrences, 19 single FAs and 2 cases of multiple FAs with benign PTs. Whole-exome sequencing was performed on one borderline PT.

Results: Recurrent PTs and multiple FAs showed temporal discordance in MED12 but not TERT. Recurrent samples did acquire TERT mutations, with recurrent benign PTs more likely to have mutations in both genes. TERT mutations were not helpful in differentiating between benign PTs and FAs in cases of multiple FAs/PTs. Exome sequencing revealed a nonsense mutation in RBM15 and Sanger sequencing revealed another three RBM15 mutations in malignant/borderline PTs.

Conclusions: This study has shown that MED12 mutations can be heterogeneous in both synchronous and recurrent PTs unlike TERT mutations. We have also shown that RBM15 mutations may be important in the pathogenesis of borderline/malignant PTs.

Phyllodes tumours (PTs) are rare fibroepithelial neoplasms of the breast composed of both stromal and epithelial elements similar to fibroadenomas (FAs) but with a more cellular stromal element. Histologically, PTs are classified as benign, borderline or malignant on the basis of stromal cellularity, nuclear atypia, mitotic activity, stromal overgrowth and type of border (infiltrating or pushing) (Lakhani et al, 2012). Borderline PTs have some but not all of the features of malignant PTs. Unlike FAs, PTs can recur locally and metastasise as sarcoma. Stromal atypia, mitoses, overgrowth and surgical margin status have been shown to be independent predictors of local recurrence (Tan et al, 2012). Most recurrent benign and borderline tumours are histologically similar to the primary neoplasms but can be more cellular, and malignant transformation has been described (Jones et al, 2008).

Recent sequencing studies have shown that MED12 exon 2 mutations occur in approximately $70 \%$ of PTs and FAs (Cani et al, 2015; Nagasawa et al, 2015; Yoshida et al, 2015a; Lien et al, 2016a). Some studies suggest that the frequency is similar across both FAs and all grades of PTs (Cani et al, 2015; Yoshida et al, 2015a; Nagasawa et al, 2015; Lien et al, 2016a) and others that they are less frequent in malignant PTs (Pfarr et al, 2015; Piscuoglio et al, 2015) and FAs ( $\mathrm{Ng}$ et al, 2015). This variation may be due to the

*Correspondence: Professor E Sawyer; E-mail: elinor.sawyer@kcl.ac.uk

Received 29 June 2017; revised 15 November 2017; accepted 16 November 2017; published online 9 January 2018

(C) 2018 Cancer Research UK. All rights reserved 0007 - 0920/18 
type of FAs included in the series as two studies have shown MED12 mutations are more common in intracanalicular than pericanalicular FAs (Yoshida et al, 2015a, b; Mishima et al, 2015). In both PTs and FAs, mutations are confined to the stromal component (Yoshida et al, 2015a, b; Mishima et al, 2015). PTs with MED12 mutations tend to have lower recurrence rates than PTs without MED12 mutations ( $\mathrm{Ng}$ et al, 2015; Yoon et al, 2016). MED12 exon 2 mutation can also help distinguish PTs from other spindle neoplasms of the breast, such as sarcomas (Lien et al, 2016b).

The finding in some studies that MED12 mutations are less frequent in malignant PTs suggests that these tumours may be driven by other genetic events. Exome and targeted sequencing studies of malignant PTs have shown that they harbour recurrent mutations in the TERT promoter, TP53, RB1, EGFR, PIK3CA, FGFR1, SETD2 and KMT2D (Tan et al, 2015; Gatalica et al, 2016; Liu et al, 2016), with TERT promoter mutations being the most frequent, occurring in $\sim 70 \%$ of malignant/borderline PTs. TERT promoter mutations are also found in benign PTs $(\sim 50 \%)$ but are not as frequent as in malignant/borderline PTs (Yoshida et al, 2015b; Piscuoglio et al, 2016). They are rare in FAs $(0-7 \%)$, suggesting that these mutations drive the progression of PTs. It has also been suggested that TERT mutations may be useful in distinguishing between benign PTs and cellular FAs (Tan et al, 2015), particularly in rare cases of multiple recurrent FAs where benign PTs have occasionally been described (Courtillot et al, 2010). TERT mutations can co-exist with MED12 mutations and similar to MED12 mutations are restricted to the stromal component (Yoshida et al, 2015b; Piscuoglio et al, 2016).

The aims of this study were to:

1. assess MED12 and TERT promoter mutations in a series of recurrent PTs;

2. assess whether TERT mutations could be helpful in distinguishing FAs from PTs in patients with synchronous/metachronous multiple FAs and PTs; and

3. identify novel mutations in other genes that may be driving malignant progression of PTs.

\section{MATERIALS AND METHODS}

Sample collection. Formalin-fixed, paraffin-embedded tissue (FFPE) was obtained from 75 primary PTs and any ipsilateral recurrences together with two cases of multiple FAs that had developed benign PTs from 15 centres across the United Kingdom with ethical approval (MREC No. 03/12/083) and informed consent. Eleven cases had paired germline DNA extracted from blood samples. H\&E-stained slides of each case were reviewed by a single histopathologist $(\mathrm{AH})$ to confirm the diagnosis and DNA was extracted as previously described (Jones et al, 2008). Nineteen FAs (FFPE) and one fresh-frozen borderline PT were provided by the KHP Cancer Biobank (NHS REC ref. 12-EE-0493).

Sanger sequencing. The promoter region of TERT was amplified by PCR as previously described (Yoshida et al, 2015b) using the following primers: $5^{\prime}$-CAGCGCTGCCTGAAACTC- $3^{\prime}$ and $5^{\prime}$ GTCCTGCCCCTTCACCTT-3'. MED12 primers, targeting exon 2, were designed with the online tool Primer3: 5'-TGTTCTACACGGAACCCTCCTC- $3^{\prime}$ and $5^{\prime}$-CTGGGCAAATGCCAATGAGAT-3'. Primers for the entire RBM15 gene followed the same design and are listed in Supplementary Table 1. Sanger sequencing was performed in a 3730xl DNA Analyser (ThermoFisher, Waltham, MA, USA) according to the manufacturer's protocol. The analysis of the electropherograms was performed in the openly available 4peaks software.
Exome sequencing. A library was prepared from tumour and paired constitutional DNA using the SureSelect Human All Exon $50 \mathrm{Mb}$ kit (Agilent, Santa Clara, CA, USA) and sequenced on Illumina HiSeq 2000 (San Diego, CA, USA) to a mean depth of $>100 \times$. Subsequent analysis was performed using our in-house pipeline; in brief, sequencing reads were aligned to the reference human genome hg19 using NovoAlign (http://www.novocraft.com/products/novoalign/), Samtools (http://www.samtools.sourceforge.net/) was used to create a pileup file and VarScan2 (http:// www.varscan.sourceforge.net/) was used to call somatic mutations and indels that were annotated using ANNOVAR (http:// annovar.openbioinformatics.org/en/latest/) and cross referenced with dbSNP and 1000 Genomes. Somatic mutations were called if there was a minimum of $30 \times$ coverage and the mutation was present in at least $10 \%$ of reads.

\section{RESULTS}

Seventy-five primary PTs (27 malignant, 22 borderline, 26 benign) were studied, of which 21 had recurred at least once (Table 1, Supplementary Table 2). Of the 21 recurrent cases, nine were benign (one of which recurred as a borderline PT), five were borderline (three of which recurred as malignant PTs) and seven were malignant. Nineteen FAs were also analysed.

Frequency of MED12 mutations. MED12 mutations occurred in $22,27,54$ and $21 \%$ of malignant, borderline and benign PTs and FAs, respectively (Table 2). MED12 mutations were more common in benign PTs compared with malignant/borderline $(P=0.02$, Fisher's Exact Test).

MED12 mutations in recurrent PTs. Although MED12 mutations appeared less common in PTs that recurred (19\%) compared with those that did not (41\%); this difference was not statistically significant ( $P=0.1$, Fisher's Exact Test; Table 2).

In the four primary tumours with MED12 mutations that did recur, two had the same $M E D 12$ mutation in the paired recurrent tumour (one had two MED12 mutations and both were seen in the recurrent sample), but in the other two cases (one malignant, one benign), there was no evidence of the original MED12 mutation in the recurrence.

There was also evidence of recurrent tumours acquiring MED12 mutations. Four cases (three benign, one borderline) with no evidence of a MED12 mutation in the primary PT did have MED12 mutations in the recurrent tumours (Table 1). Similarly, in three cases that developed a second recurrence, none had a MED12 mutation in the primary, two had a MED12 mutation in the first recurrence, and of these, one had the same MED12 mutation in the second recurrence and the other a different MED12 mutation.

Multiple MED12 mutations in the same lesion. We also found evidence of multiple MED12 mutations in the same lesions (Supplementary Table 3). Five primary PTs (one of which recurred and both mutations were found in the recurrence) and another two recurrences had multiple (2-4) MED12 mutations. Of these two recurrences, one had no evidence of MED12 mutations in the primary and the other had a single MED12 mutations in the primary lesion and then acquired another three mutations in the recurrence. All cases with multiple MED12 mutations were benign.

Frequency of TERT promoter mutations. TERT promoter mutations occurred in 48,55, 31 and $0 \%$ of malignant, borderline, and benign PTs and FAs, respectively (Table 2). There was no significant difference in the frequency of TERT mutations between the different subtypes of PT, but as expected a clear difference between PTs and FAs ( $P=0.0001$, Fisher's Exact Test). 
Table 1. Detailed table of recurrences and their MED12 and TERT promoter mutations

\begin{tabular}{|c|c|c|c|c|c|c|c|}
\hline $\begin{array}{l}\text { Sample } \\
\text { ID }\end{array}$ & Gene & $\begin{array}{l}\text { Primary } \\
\text { subtype }\end{array}$ & Primary mutation & $\begin{array}{l}\text { Recurrence } 1 \\
\text { subtype }\end{array}$ & Recurrence 1 mutation & $\begin{array}{l}\text { Recurrence } 2 \\
\text { subtype }\end{array}$ & $\begin{array}{c}\text { Recurrence } 2 \\
\text { mutation }\end{array}$ \\
\hline 1 & TERT & $M$ & c. $-124 C>T$ & $M$ & c. $-124 \mathrm{C}>\mathrm{T}$ & & \\
\hline 2 & TERT & M & No & M & No & & \\
\hline 3 & MED12 & M & No & M & No & M & No \\
\hline \multirow[t]{2}{*}{4} & MED12 & M & c. $131 \mathrm{G}>\mathrm{A}$ & M & No & & \\
\hline & TERT & M & No & M & No & & \\
\hline \multirow[t]{2}{*}{5} & MED12 & M & No & M & No & & \\
\hline & TERT & M & No & M & No & & \\
\hline 6 & MED12 & $M$ & No & $\mathrm{M}$ & No & & \\
\hline \multirow[t]{2}{*}{8} & MED12 & Bo & No & M & No & & \\
\hline & TERT & Bo & c. $-124 C>T$ & M & c. $-124 C>T$ & & \\
\hline \multirow[t]{2}{*}{9} & MED12 & Bo & No & M & c. $100-13 T>C$ & & \\
\hline & TERT & Bo & No & M & No & & \\
\hline \multirow[t]{2}{*}{10} & MED12 & Bo & No & M & No & & \\
\hline & TERT & Bo & No & $M$ & c. $-124 C>T$ & & \\
\hline \multirow[t]{2}{*}{11} & MED12 & Bo & No & Bo & No & & \\
\hline & TERT & Bo & No & Bo & No & & \\
\hline \multirow[t]{2}{*}{12} & MED12 & Bo & No & Bo & No & & \\
\hline & TERT & Bo & No & Bo & No & & \\
\hline \multirow[t]{2}{*}{16} & MED12 & B & No & Bo & No & & \\
\hline & TERT & B & No & Bo & No & & \\
\hline \multirow[t]{2}{*}{17} & MED12 & B & c. $131 \mathrm{G}>\mathrm{C}$ & B & $\begin{array}{c}\text { c.128A }>\text { C, } 129 \mathrm{~A}>\mathrm{G}, \mathrm{c} .131 \mathrm{G}>\mathrm{C} \\
\text { c.133_152del30 }\end{array}$ & & \\
\hline & TERT & B & c. $-124 C>T$ & B & c. $-124 C>T$ & & \\
\hline \multirow[t]{2}{*}{18} & MED12 & B & No & B & c.120_146del27 & B & c.113_151del39 \\
\hline & TERT & B & c. $-124 C>T$ & B & c. $-124 C>T$ & $B$ & c. $-124 C>T$ \\
\hline \multirow[t]{2}{*}{19} & MED12 & B & No & B & c.122_148_del27 & B & c.122_148_del27 \\
\hline & TERT & B & c. $-124 C>T$ & $B$ & c. $-124 \mathrm{C}>\mathrm{T}$ & $B$ & c. $-124 \mathrm{C}>\mathrm{T}$ \\
\hline \multirow[t]{2}{*}{20} & MED12 & B & c.124_156del33 & $B$ & No & & \\
\hline & TERT & B & No & B & No & & \\
\hline \multirow[t]{2}{*}{21} & MED12 & B & No & B & c.148G > A and c.133_147del15 & & \\
\hline & TERT & B & No & B & c. $-124 \mathrm{C}>\mathrm{T}$ & & \\
\hline
\end{tabular}

TERT promoter mutations in recurrent PTs. Similar to MED12, there was no evidence that TERT promoter mutations were more or less common in cases that recurred (28\% recurrent vs $50 \%$ non-recurrent). However unlike MED12 all seven of the primary PTs with a TERT promoter mutation that recurred showed evidence of the mutation in the paired recurrence. Of those, three (benign) had a second recurrence, which also had evidence of the mutation.

There was also evidence of recurrent tumours acquiring TERT promoter mutations with four (one malignant, one borderline and two benign) acquiring a TERT promoter mutation in the first recurrence and again this was also found in subsequent recurrences (Table 1). 
Table 2. Frequency of MED12 and TERT promoter mutations in recurrent and non-recurrent phyllodes tumours and fibroadenomas

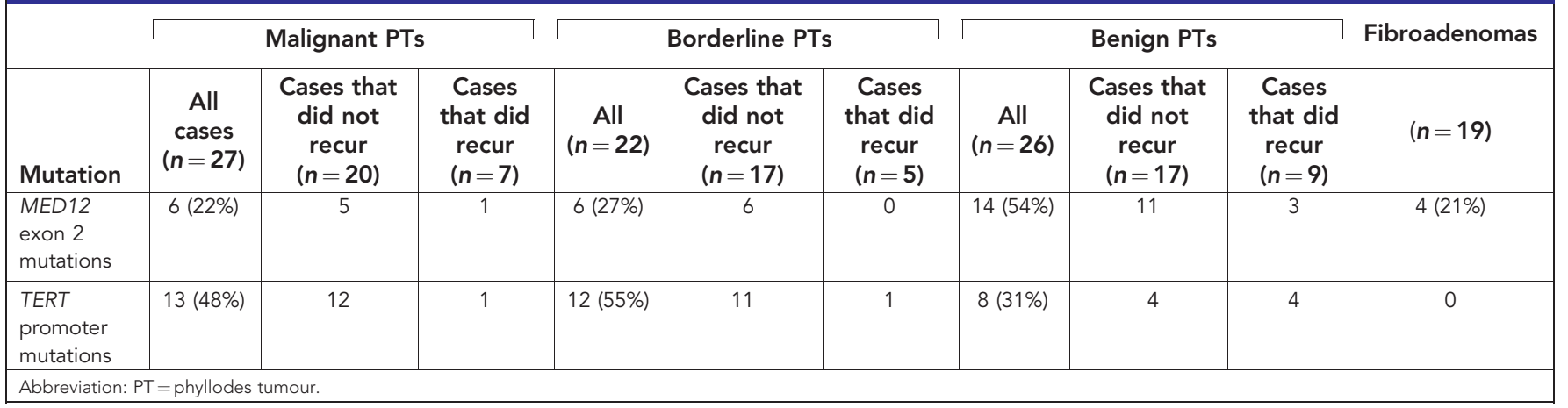

\section{Table 3. Somatic mutations identified by whole exome sequencing of a single borderline phyllodes tumour}

\begin{tabular}{|c|c|c|c|c|}
\hline Chrom & Gene & Site of mutation & Annotation & $\%$ of reads with mutation \\
\hline chr1 & RBM15 & Exonic & RBM15:NM_001201545:exon1:c.A583T:p.K195X: stopgain & $64.20 \%$ \\
\hline chr1 & VASH2 & Splicing & VASH2:NM_024749:exon4:c.366-1G >C & $61.54 \%$ \\
\hline chr11 & $\mathrm{ROBO} 3$ & Splicing & ROBO3:NM_022370:exon12:c.1785-4G > T & $44.44 \%$ \\
\hline chr11 & TRIM49C & Exonic & TRIM49C:NM_001195234:exon3:c.T185C:p.I62T & $41.63 \%$ \\
\hline chr3 & CAND2 & Exonic & CAND2:NM_001162499:exon10:c.A2030G:p.D677G & $40.23 \%$ \\
\hline chr15 & PLA2G4F & Exonic & PLA2G4F:NM_213600:exon1:c.27delG: frameshift deletion & $37.88 \%$ \\
\hline chr2 & NEB & Exonic & NEB:NM_001164507:exon37:c.G4182T:p.K1394N & $35.97 \%$ \\
\hline chr10 & ENKUR & Exonic & ENKUR:NM_145010:exon2:c.T198A:p.H66Q, & $35.26 \%$ \\
\hline chr1 & YIPF1 & Exonic & YIPF1:NM_018982:exon4:c.T89C:p.I30T, & $34.45 \%$ \\
\hline chr5 & PCDHA11 & Exonic & PCDHA11:NM_031861:exon1:c.C1446A:p.D482E & $32.03 \%$ \\
\hline $\operatorname{chr} X$ & FAM58A & Exonic & Unknown & $21.86 \%$ \\
\hline chr12 & LIMA1 & Splicing & LIMA1:NM_016357:exon9:c.973-3G > - & $20.83 \%$ \\
\hline chr1 & CACNA1S & Splicing & CACNA1S:NM_000069:exon5:c.399-6T>C & $17.43 \%$ \\
\hline chr15 & ZNF280D & Splicing & ZNF280D:NM_017661:exon11:c.781-8C > - & $16.28 \%$ \\
\hline chr14 & RALGAPA1 & Splicing & RALGAPA1:NM_014990:exon11:c.1012-8G>-, & $15.58 \%$ \\
\hline chr19 & SSC5D & Exonic & SSC5D:NM_001144950:exon14:c.T4361C:p.L1454P & $15.38 \%$ \\
\hline chr11 & BRSK2 & Exonic & BRSK2:NM_001256630:exon1:c.A17C:p.H6P, & $15.15 \%$ \\
\hline chr20 & BCAS4 & Exonic & BCAS4:NM_198799:exon2:c.T191G:p.V64G & $15 \%$ \\
\hline
\end{tabular}

Analysis of two cases of multiple FAs and PTs. In order to assess whether TERT promoter mutations could be useful in distinguishing between benign PTs and FAs in patients with multiple FAs, we analysed cases from two young women who developed bilateral multiple recurrent FAs and subsequently developed benign PTs (Supplementary Figure 1). The first, aged 22 years, developed multiple bilateral FAs over a period of 3 years and was diagnosed with benign PTs on the fourth recurrence. Nine lesions (7 FAs and 2 PTs) were examined from the 4 -year period and none showed any evidence of TERT mutations. At the fourth recurrence, MED12 mutations were identified in one cellular FA (c.130G > A) and one benign PT (c.130G $>$ A and c.136_150del15). The second case occurred in a 20-year-old with bilateral multiple FAs that started to increase in size and so were excised and found to be a cellular FA and two benign PTs. Sequencing of each lesion showed no TERT mutations and a different MED12 mutation in each of the lesions (c.107T $>$ G, c.131G $>$ A and c.100-8T $>$ A).

Novel drivers of malignant and borderline PTs. In an attempt to identify other drivers of malignant and borderline PTs, we performed whole-exome sequencing of a single borderline case who had DNA available from fresh-frozen tumour and blood. Eighteen somatic mutations were identified but no MED12 mutation (Table 3). The mutations were ranked according to the variant allele frequency within the tumour, on the assumption that those with a frequency of $\sim 50 \%$ were less likely to be subclonal and more likely to be driver mutations. Five mutations with a variant frequency of $40-64 \%$ were identified and verified by Sanger sequencing in DNA extracted from FFPE material from the same tumour. Only one of these genes, RBM15, had previously been identified as harbouring a mutation in a PT (Tan et al, 2015; borderline PT with a frameshift RBM15 mutation, c.598_601delGTAA). We therefore chose to Sanger sequence this gene in another 27 malignant, 17 borderline (including one recurrent sample) and 16 benign (including five recurrent samples) and found another three different mutations, two in malignant PTs and one in a borderline PT (Table 4, Figure 1, Supplementary Figure 2).

\section{DISCUSSION}

MED12 and TERT promoter mutations have previously been shown to be the most common mutations in PTs. This study, similar to others (Table 5A), has shown that MED12 mutations are more common in benign PTs than malignant and borderline PTs 
Table 4. RBM15 mutations

\begin{tabular}{|c|c|c|c|c|c|}
\hline Sample ID & Histopath subtype & Mutation DNA & Mutation AA & DANN $^{\mathrm{a}}$ score & CADD $^{\mathrm{b}}$ score \\
\hline 22 & Borderline & c. $583 \mathrm{~A}>\mathrm{T}$ & $\mathrm{p} .195 \mathrm{~K}>^{\mathrm{a}}$ & 0.999 & 26.3 \\
\hline 23 & Malignant & c. $1924 \mathrm{C}>\mathrm{T}$ & $p .642 R>^{a}$ & 0.995 & 36 \\
\hline 24 & Borderline & c.715delG & p. $239 \mathrm{~V}>\mathrm{fs}^{\mathrm{a}} 1$ & NA (del) & NA (del) \\
\hline 25 & Malignant & c. $2344 \mathrm{C}>\mathrm{T}$ & p.782P $>S$ & 0.886 & 11.13 \\
\hline \multicolumn{6}{|c|}{$\begin{array}{l}\text { Abbreviations: NA }=\text { not applicable. } \\
{ }^{a} \text { DANN (https://doi.org/10.1093/bioinformatics/btu703) uses a } 0-1 \text {, scale. Score } \geqslant 0.96 \text { identifies } 92 \% \text { of the true positive pathogenic variations, with } 18.1 \% \text { false positive benign variations. } \\
{ }^{b} \text { CADD (cadd.gs.washington.edu/). A scaled CADD score of } 20 \text { means that a variant is among the top } 1 \% \text { of deleterious variants in the human genome. Scaled CADD score of } 30 \text { means that the } \\
\text { variant is in the top } 0.1 \% \text {. }\end{array}$} \\
\hline
\end{tabular}

and TERT promoter mutations are rare in FAs. By analysing paired primaries and recurrences, we have been able to assess whether these mutations are also present in paired recurrences or whether there is evidence of temporal heterogeneity. We have demonstrated that $M E D 12$ is frequently heterogeneous between lesions from the same patient, in contrast to TERT promoter mutation that are consistently found in paired recurrences, including those with multiple recurrences. Lae et al (2016) also demonstrated temporal heterogeneity within MED12 (they did not assess TERT). In their study, the heterogeneous recurrences had different MED12 mutations from the primary case, this occurred in one of our recurrent cases (a second benign recurrence) but the remainder (one benign, one malignant) lacked a MED12 mutation in the paired recurrent sample, suggesting that they were either new primaries or had arisen from a subclone within the primary PT that did not contain the MED12 mutation. Unlike Lae et al (2016), we also have evidence of four cases of wild-type MED12 primaries acquiring MED12 mutations in the second event (three benign, one borderline) and of benign PTs harbouring multiple (2-4) MED12 mutations in the same lesion. As Sanger sequencing will only detect clonal mutations, we cannot exclude the possibility that it was a subclonal mutation in the primary tumour which we cannot detect. These findings suggest that MED12 are not only just early events in fibroepithelial tumours but also provide some growth advantage in established benign PTs.

A number of recurrences also acquired TERT promoter mutations, ( 4 cases -2 benign and 2 malignant). In the series of PTs described by Yoshida et al 2015b; TERT and MED12 mutations were frequently found together (Table $5 \mathrm{~B}$ ). We did not see this in the primary cases, $41 \%$ of PTs with TERT promoter mutations harboured MED12 mutations, which is similar to (Piscuoglio et al, 2016) (52\%) and (Liu et al, 2016) (50\%). However, in the recurrent samples TERT and MED12 mutations frequently co-occured $(P=0.05$, Fisher's Exact test), particularly in benign cases where acquisition of MED12 and TERT promoter mutations resulted in six out of nine benign recurrences having both a TERT and MED12 mutation compared with one out of nine of their paired primary samples. In contrast, none of the malignant/borderline recurrences had mutations in both genes. This suggests that although they can co-exist TERT promoter mutations are not dependent on MED12 mutations. As postulated by (Piscuoglio et al, 2016) TERT promoter mutations may allow the stroma of PTs to undergo more cycles of cell division and thus increase the chance of them acquiring a driver mutation.

The lack of TERT promoter mutations in FAs has led some authors to suggest that this mutation may be useful for distinguishing between FAs and benign PTs in rare cases of multiple FAs and benign PTs. In two such cases, we found no evidence of TERT promoter mutations in either the FAs or benign PTs. The analysis of these multiple tumour cases once again demonstrated heterogeneity of MED12. In the first case, no MED12 mutation was present in the initial FAs and only appeared at the time of the fourth recurrence when a cellular FA and benign PT were diagnosed on histology - the presence of the same mutation suggested a common clonal origin, although the PT had also acquired a second MED12 mutation. In contrast, in the second case (a cellular FA and two benign PTs), all three lesions had different MED12 mutations, suggesting that they arose independently (Supplementary Figure 1).

The mechanism through which MED12 mutations confer a growth advantage to FAs and benign PTs is not clear. MED12 mutations are also frequent in uterine leiomyomas but less so in uterine leiomyosarcomas (Ravegnini et al, 2013). A possible explanation for this is that MED12 mutations drive benign proliferation of smooth muscle and stroma in the uterus and breast, respectively, resulting in leiomyomas and FAs/benign PTs. In rare cases, these benign lesions progress to leiomyosarcoma and malignant PTs, but the majority of these malignant tumours arise de novo and therefore do not have MED12 mutations.

The exome sequencing of the single borderline PT with freshfrozen tissue did not reveal any MED12 mutation and no TERT promoter mutation was found on Sanger Sequencing, but it did identify mutations in 18 genes, none of which had been previously identified as driver mutations in any type of cancer. The most frequent variant in this PT was a nonsense mutation in $R B M 15$, an RNA-binding protein on 1 p13.3 involved in regulating splicing of GATA1 and RUNX transcription factors in megakaryocyte differentiation and translocated in infant acute megakaryocytic leukaemia (Tran et al, 2016). Interestingly, previously published array CGH data of this tumour (Jones et al, 2008) showed loss in this region (1:105666811-117575143, GRCh37/ hg19), Supplementary Figure 3.

A frameshift mutation (c.598_601delGTAA) in RBM15 was also detected by exome sequencing by (Tan et al, 2015) in a borderline PT but was not remarked upon as a possible driver gene. All other PT sequencing studies have used targeted sequencing panels that have not included $R B M 15$. Sanger sequencing of our tumour series identified a further three RBM15 mutations in malignant/borderline PTs, two of which were nonsense or frameshift changes and one of which was a missense mutation, not predicted to be deleterious but located at the start of a highly conserved C-terminal SPOC (Spen paralog and ortholog C-terminal) domain. One hundred and sixty-six mutations in RBM15 have been catalogued in COSMIC (http://cancer.sanger.ac.uk/cosmic), of which only 10 are nonsense and occur in a variety of solid tumours, including bladder, head and neck, colorectal, stomach, prostate and pancreas. As well as being involved in the development of megakaryocytic leukaemia, RBM15 is an important factor in $\mathrm{X}$ chromosome silencing (Moindrot et al, 2015) and has been shown to be expressed in mammary tissue (https://www.gtexportal.org/home/). It is therefore not unreasonable to suggest that a truncated or absent RBM15 protein may confer a growth advantage in PTs.

Of the five RBM15 mutations that have now been described in PTs (four in this study, one by Tan et al, 2015), three occurred in malignant/borderline PTs that did not harbour MED12 or TERT promoter mutations. Previous literature also suggests that PIK3CA mutations are more common in PTs that do not harbour MED12 mutations (Tan et al, 2015; Piscuoglio et al, 2016). In 


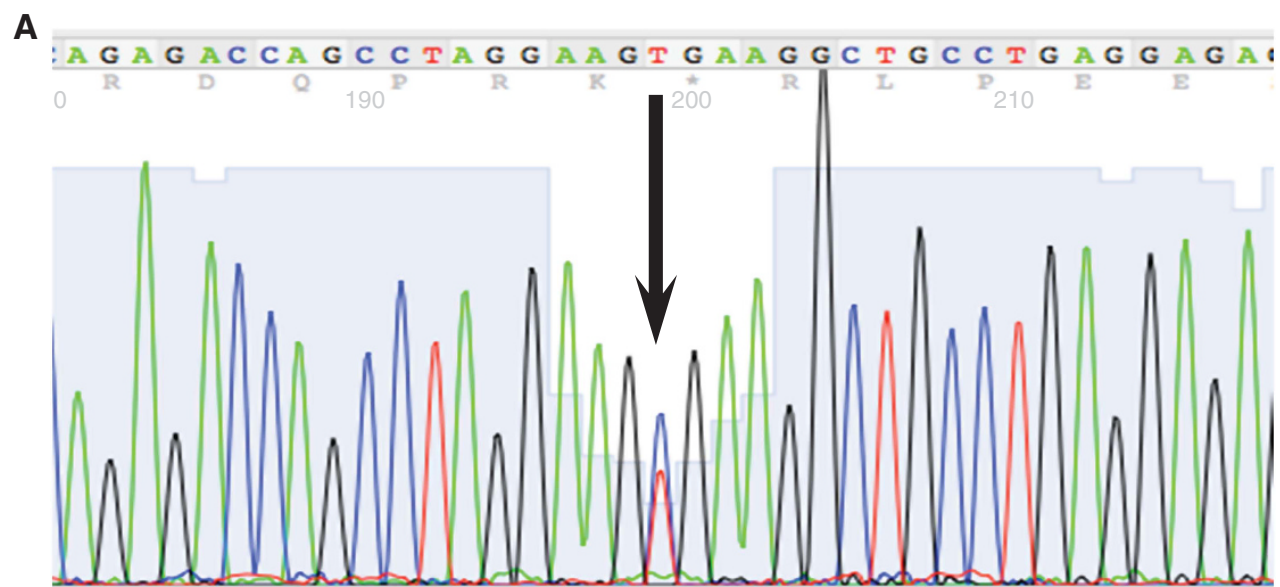

B
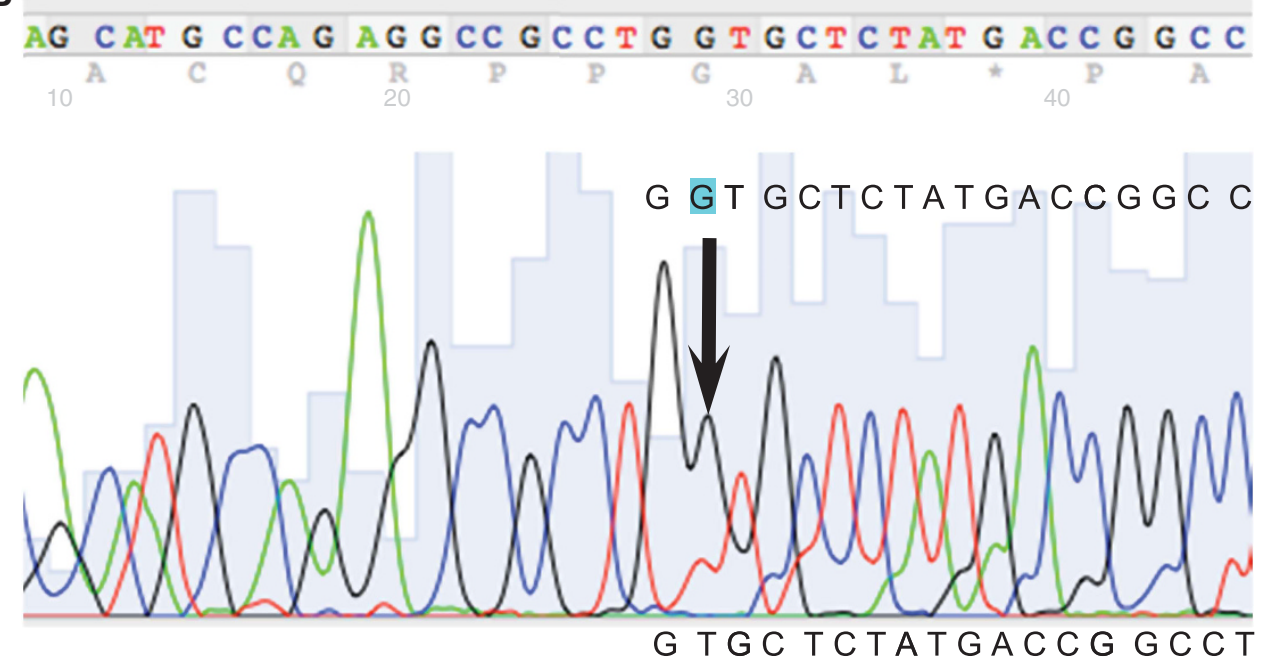

C

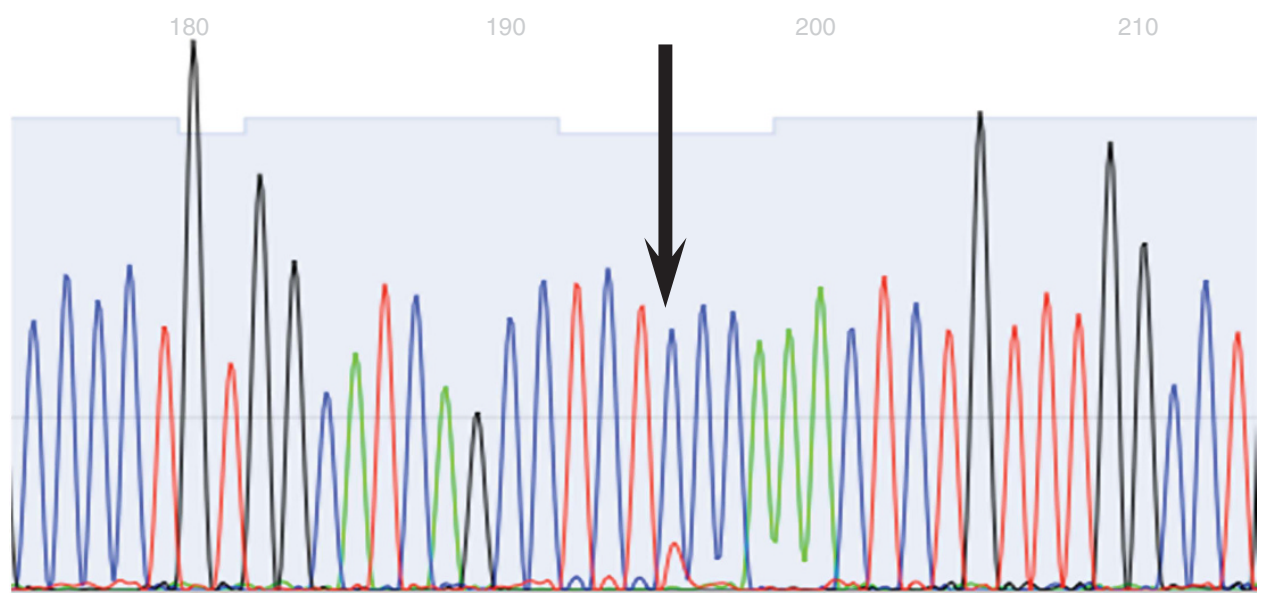

Figure 1. RBM15 mutations identified by Sanger sequencing. (A) Sample 23 (c.1924 C>T, p.R642*). (B) Sample 24 (c.715delG, p.V239fs`1) correct sequence shown above, shifted sequence shown below (reverse sequence shown in Supplementary Figure 2). (C) Sample 25 (c.2344C $>$ T, p.P782S).

contrast, RARA mutations were more frequent in samples with MED12 and TERT promoter mutations (Tan et al, 2015; Piscuoglio et $a l, 2016)$ and thus may provide a selective advantage when TERT/MED12 are mutated.

In conclusion, we have shown that, although MED12 mutations are common in both benign PTs and FAs, suggesting that they are sometimes early events in fibroepithelial lesions of the breast, they can be discordant in recurrent PTs, particularly in benign cases where they can be lost or acquired with some cases carrying multiple mutations in MED12. There was less evidence of temporal heterogeneity in TERT promoter mutations, but recurrent samples did acquire TERT promoter mutations, supporting previous data from our laboratory that recurrent samples often acquired new genetic changes (Jones et al, 2008).

Through exome sequencing of a single malignant PT, we have shown that RBM15 may be a novel driver mutation in malignant/ 
Table 5A. Summary of previous published data on MED12 mutations in FAs and PTs

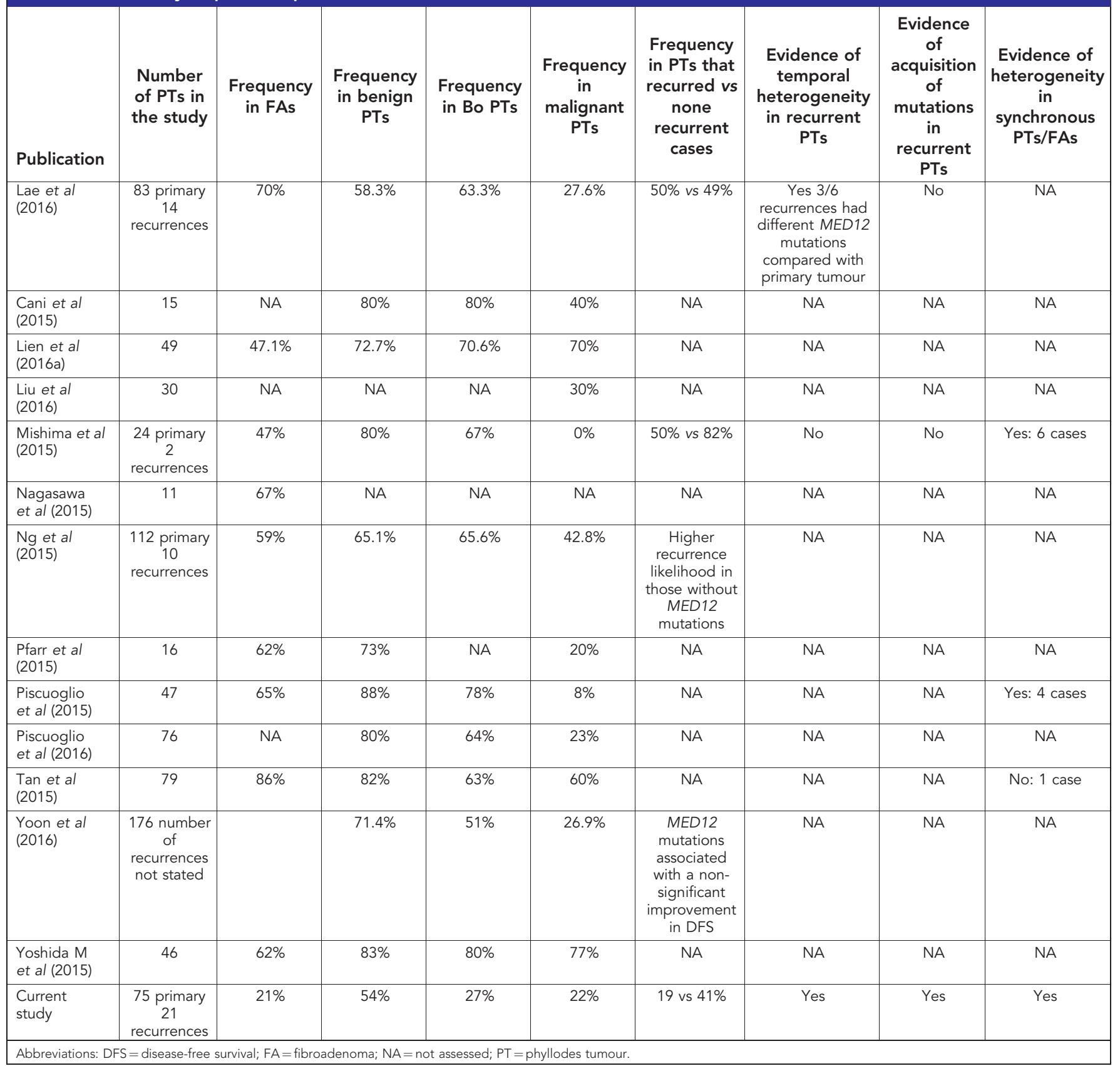

\section{Table 5B. Summary of previous published data on TERT promoter mutations in FAs and PTs}

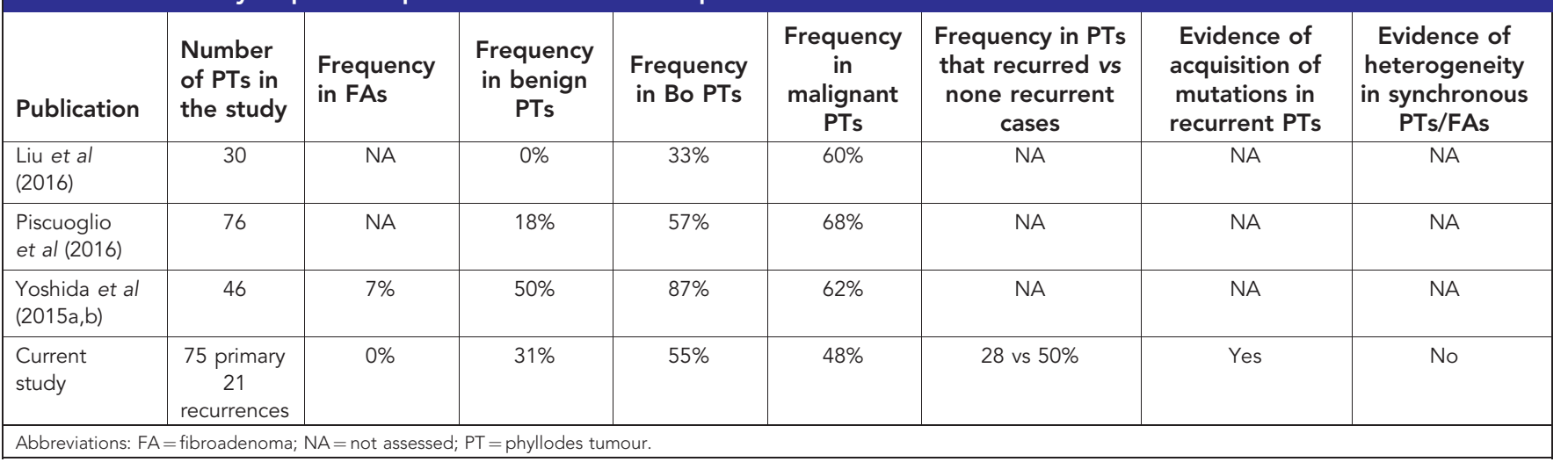


borderline PTs at a frequency of $7 \%$, but this requires further validation in additional sample sets. In order to identify other drivers of malignant PTs, further analysis of these unusual tumours would be better carried out through exome or whole-genome sequencing rather than targeted sequencing in order to detect mutations in genes not currently known to be drivers of solid cancers.

\section{ACKNOWLEDGEMENTS}

Fibroadenoma material and fresh-frozen material were provided by King's Health Partners Cancer Biobank, London, UK, which is supported by the Experimental Cancer Medicine Centre at King's College London and the Department of Health via the National Institute for Health Research Comprehensive Biomedical Research Centre Award. This research was funded by a Department of Health Clinician Scientist Grant and the National Institute for Health Research (NIHR) Biomedical Research Centre based at Guy's and St. Thomas' NHS Foundation Trust and King's College London.

\section{CONFLICT OF INTEREST}

The authors declare no conflict of interest.

\section{REFERENCES}

Cani AK, Hovelson DH, McDaniel AS, Sadis S, Haller MJ, Yadati V, Amin AM, Bratley J, Bandla S, Williams PD, Rhodes K, Liu CJ, Quist MJ, Rhodes DR, Grasso CS, Kleer CG, Tomlins SA (2015) Next-gen sequencing exposes frequent $M E D 12$ mutations and actionable therapeutic targets in phyllodes tumors. Mol Cancer Res 13(4): 613-619.

Courtillot C, Chakhtoura Z, Bogorad R, Genestie C, Bernichtein S, Badachi Y, Janaud G, Akakpo JP, Bachelot A, Kuttenn F, Goffin V, Touraine P. Benign Breast Diseases Study Group (2010) Characterization of two constitutively active prolactin receptor variants in a cohort of 95 women with multiple breast fibroadenomas. J Clin Endocrinol Metab 95(1): 271-279.

Gatalica Z, Vranic S, Ghazalpour A, Xiu J, Ocal IT, McGill J, Bender RP, Discianno E, Schlum A, Sanati S, Palazzo J, Reddy S, Pockaj B (2016) Multiplatform molecular profiling identifies potentially targetable biomarkers in malignant phyllodes tumors of the breast. Oncotarget 7(2): 1707-1716.

Jones AM, Mitter R, Springall R, Graham T, Winter E, Gillett C, Hanby AM, Tomlinson IP, Sawyer EJ. Phyllodes Tumour Consortium (2008) A comprehensive genetic profile of phyllodes tumours of the breast detects important mutations, intra-tumoral genetic heterogeneity and new genetic changes on recurrence. J Pathol 214(5): 533-544.

Laé M, Gardrat S, Rondeau S, Richardot C, Caly M, Chemlali W, Vacher S, Couturier J, Mariani O, Terrier P, Bièche I (2016) MED12 mutations in breast phyllodes tumors: evidence of temporal tumoral heterogeneity and identification of associated critical signaling pathways. Oncotarget 7(51): 84428-84438.

Lakhani SR, Ellis IO, Schnitt SJ, Tan PH, van de Vijver MJ (2012) World Health Organization Classification of Tumours of the Breast. IARC: Lyon, France.

Lien HC, Huang CS, Yang YW, Jeng YM (2016a) Mutational analysis of MED12 exon 2 in a spectrum of fibroepithelial tumours of the breast: implications for pathogenesis and histogenesis. Histopathology 68(3): 433-441.

Lien HC, Huang CS, Yang YW, Jeng YM (2016b) MED12 exon 2 mutation as a highly sensitive and specific marker in distinguishing phyllodes tumours from other spindle neoplasms of the breast. APMIS 124(5): 356-364.

Liu SY, Joseph NM, Ravindranathan A, Stohr BA, Greenland NY, Vohra P, Hosfield E, Yeh I, Talevich E, Onodera C, Van Ziffle JA, Grenert JP,
Bastian BC, Chen YY, Krings G (2016) Genomic profiling of malignant phyllodes tumors reveals aberrations in FGFR1 and PI-3 kinase/RAS signaling pathways and provides insights into intratumoral heterogeneity. Mod Pathol 29(9): 1012-1027.

Mishima C, Kagara N, Tanei T, Naoi Y, Shimoda M, Shimomura A, Shimazu K, Kim SJ, Noguchi S (2015) Mutational analysis of MED12 in fibroadenomas and phyllodes tumors of the breast by means of targeted next-generation sequencing. Breast Cancer Res Treat 152(2): 305-312.

Moindrot B, Cerase A, Coker H, Masui O, Grijzenhout A, Pintacuda G, Schermelleh L, Nesterova TB, Brockdorff N. (2015) A pooled shRNA screen identifies Rbm15, Spen, and Wtap as factors required for Xist RNAMediated silencing. Cell Rep 12(4): 562-572.

Nagasawa S, Maeda I, Fukuda T, Wu W, Hayami R, Kojima Y, Tsugawa K, Ohta T (2015) MED12 exon 2 mutations in phyllodes tumors of the breast. Cancer Med 4: 1117-1121.

Ng CC, Tan J, Ong CK, Lim WK, Rajasegaran V, Nasir ND, Lim JC, Thike AA, Salahuddin SA, Iqbal J, Busmanis I, Chong AP, Teh BT, Tan PH (2015) MED12 is frequently mutated in breast phyllodes tumours: a study of 112 cases. J Clin Pathol 68(9): 685-691.

Pfarr N, Kriegsmann M, Sinn P, Klauschen F, Endris V, Herpel E, Muckenhuber A, Jesinghaus M, Klosterhalfen B, Penzel R, Lennerz JK, Weichert W, Stenzinger A (2015) Distribution of MED12 mutations in fibroadenomas and phyllodes tumors of the breast-implications for tumor biology and pathological diagnosis. Genes Chromosomes Cancer 54(7): 444-452.

Piscuoglio S, Murray M, Fusco N, Marchiò C, Loo FL, Martelotto LG, Schultheis AM, Akram M, Weigelt B, Brogi E, Reis-Filho JS (2015) MED12 somatic mutations in fibroadenomas and phyllodes tumours of the breast. Histopathology 67(5): 719-729.

Piscuoglio S, Ng CK, Murray M, Burke KA, Edelweiss M, Geyer FC, Macedo GS, Inagaki A, Papanastasiou AD, Martelotto LG, Marchio C, Lim RS, Ioris RA, Nahar PK, Bruijn ID, Smyth L, Akram M, Ross D, Petrini JH, Norton L, Solit DB, Baselga J, Brogi E, Ladanyi M, Weigelt B, Reis-Filho JS (2016) Massively parallel sequencing of phyllodes tumours of the breast reveals actionable mutations, and TERT promoter hotspot mutations and TERT gene amplification as likely drivers of progression. J Pathol 238(4): 508-518.

Ravegnini G, Mariño-Enriquez A, Slater J, Eilers G, Wang Y, Zhu M, Nucci MR, George S, Angelini S, Raut CP, Fletcher JA. MED12 mutations in leiomyosarcoma and extrauterine leiomyoma. Mod Pathol (2013) 26: 743-749.

Tan J, Ong CK, Lim WK, Ng CC, Thike AA et al. (2015) Genomic landscapes of breast fibroepithelial tumors. Nat Genet 47(11): 1341-1345.

Tan PH, Thike AA, Tan WJ, Thu MM, Busmanis I, Li H, Chay WY, Tan MH, Phyllodes Tumour Network Singapore (2012) Predicting clinical behaviour of breast phyllodes tumours: a nomogram based on histological criteria and surgical margins. J Clin Pathol 65(1): 69-76.

Tran NT, Su H, Khodadadi-Jamayran A, Lin S, Zhang L, Zhou D, Pawlik KM, Townes TM, Chen Y, Mulloy JC, Zhao X. (2016) The AS-RBM15 lncRNA enhances $R B M 15$ protein translation during megakaryocyte differentiation. EMBO Rep 17(6): 887-900.

Yoon N, Bae GE, Kang SY, Choi MS, Hwang HW, Kim SW, Lee JE, Nam SJ, Gong G, Lee HJ, Bae YK, Lee A, Cho EY (2016) Frequency of MED12 mutations in phyllodes tumors: Inverse correlation with histologic grade. Genes Chromosomes Cancer 55(6): 495-504.

Yoshida M, Sekine S, Ogawa R, Yoshida H, Maeshima A, Kanai Y, Kinoshita T, Ochiai A (2015a) Frequent MED12 mutations in phyllodes tumours of the breast. Br J Cancer 112(10): 1703-1708.

Yoshida M, Ogawa R, Yoshida H, Maeshima A, Kanai Y, Kinoshita T, Hiraoka N, Sekine S (2015b) TERT promoter mutations are frequent and show association with MED12 mutations in phyllodes tumors of the breast. Br J Cancer 113(8): 1244-1248.

This work is published under the standard license to publish agreement. After 12 months the work will become freely available and the license terms will switch to a Creative Commons AttributionNonCommercial-Share Alike 4.0 Unported License. 